\title{
Breakdown of Counterflow Superfluidity in a Disordered Quantum Hall Bilayer
}

\author{
D. K. K. Lee, ${ }^{1}$ P. R. Eastham, ${ }^{2}$ and N. R. Cooper ${ }^{3}$ \\ ${ }^{1}$ Blackett Laboratory, Imperial College London, London SW7 2AZ, UK \\ ${ }^{2}$ School of Physics, Trinity College, Dublin 2, Ireland \\ ${ }^{3}$ Cavendish Laboratory, University of Cambridge, Cambridge CB3 OHE, UK
}

Correspondence should be addressed to D. K. K. Lee, dkk.lee@imperial.ac.uk

Received 1 September 2010; Accepted 12 January 2011

Academic Editor: Yogesh Joglekar

Copyright $\odot 2011$ D. K. K. Lee et al. This is an open access article distributed under the Creative Commons Attribution License, which permits unrestricted use, distribution, and reproduction in any medium, provided the original work is properly cited.

\begin{abstract}
We present a theory for the regime of coherent interlayer tunneling in a disordered quantum Hall bilayer at total filling factor one, allowing for the effect of static vortices. We find that the system consists of domains of polarized superfluid phase. Injected currents introduce phase slips between the polarized domains which are pinned by disorder. We present a model of saturated tunneling domains that predicts a critical current for the breakdown of coherent tunneling that is extensive in the system size. This theory is supported by numerical results from a disordered phase model in two dimensions. We also discuss how our picture might be used to interpret experiments in the counterflow geometry and in two-terminal measurements.
\end{abstract}

\section{Introduction}

In a quantum Hall bilayer at total Landau level filling $v_{T}=$ 1 , Coulomb interactions induce a state with interlayer phase coherence $[1,2]$. This state is expected to be approximately the Halperin [111] state [3], which can be understood as a Bose-Einstein condensate of interlayer excitons $[4,5]$. The motion of excitons corresponds to counterflowing electrical currents in the layers so that excitonic supercurrents can give dissipationless electrical transport. The superfluid properties of the [111] state have been demonstrated theoretically by Wen and Zee $[6,7]$.

This counterflow superfluidity can be probed in tunneling experiments. In the tunneling geometry (Figure 1), a current $I_{t}$ is injected into the top layer at one corner and removed from the bottom layer at the opposite corner. These current flows may be written as superpositions of layersymmetric and layer-antisymmetric currents

$$
I_{\text {in }(\text { out })}=\frac{1}{2} I_{t}\left[\left(\begin{array}{l}
1 \\
1
\end{array}\right) \pm\left(\begin{array}{c}
1 \\
-1
\end{array}\right)\right],
$$

where the two components refer to currents in the two layers. Thus, the tunneling experiment corresponds to a flow of layer-symmetric current, with equal counterflow currents $I_{\mathrm{CF}}=I_{t} / 2$ injected by both the electron source and drain. The symmetric component is transported by a dissipationless edge state, which does not penetrate the bulk due to an energy gap to charged excitations. However, the bulk can carry the counterflow component as a chargeneutral excitonic supercurrent. Since both these channels are dissipationless, we expect dissipationless electrical transport. In particular, a finite interlayer current $I$ at negligible interlayer voltage $V$ has been predicted $[8,9]$. This has been recently confirmed by four-terminal measurements by Tiemann and coworkers $[10,11]$. This phenomenon can be regarded as a form of the Josephson effect [7]. Note that thermally activated quasiparticles and contact effects [12] can give rise to complications in actual experiments.

The Josephson-like regime persists for interlayer currents up to a critical value $I_{c}$. Above $I_{c}$, interlayer transport becomes dissipative. Nevertheless, interlayer coherence can still be detected in the interlayer $I V$ characteristics of the system. A strong peak is observed at zero bias in the differential interlayer conductivity. This is followed at low bias by a regime with negative differential conductivity $[13,14]$. This regime can be studied theoretically treating the interlayer tunneling as a perturbation [15-17]. 


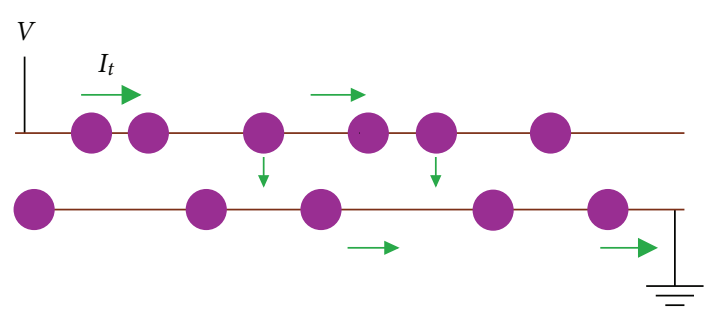

FIgURE 1: Schematic diagram of tunneling experiment.

In this paper, we focus on the Josephson regime below the critical current and present a physical picture of its breakdown. We have previously presented, in a short paper [18], a theory of this breakdown based on numerical results on a one-dimensional model. The aim of the present paper is to present numerical results for a two-dimensional model, which directly demonstrate the breakdown mechanism in a realistic geometry. The key motivation of our work is to understand the observation [11] that the critical current $I_{c}$ is proportional to the sample area. (Area scaling is also observed in the zero-bias peak of the interlayer conductivity [19]. We will discuss this in Section 6.) The source and drain contacts for the applied current are located at opposite ends of the system. If one models this system as a clean homogeneous bilayer using reasonable estimates of the tunnel splitting, one finds that the injected current should have tunneled across the bilayer within a few microns of the source contact $\left(\lambda_{J}\right.$ in (6)). Such a current profile would suggest that the critical value of the interlayer current should not depend on the sample length in the direction of the current $[12,20,21]$. Put another way, the area scaling of the critical current could only be explained by a clean model of the bilayer if one accepts a tunnel splitting that is anomalously small by several orders of magnitude [12].

A similar puzzle is found in the original observation of dissipationless counterflow $[22,23]$ in the counterflow geometry (Figure 3). Again, counterflow currents apparently traverse the system over distances orders of magnitude further than expected. We will return to this geometry in the final section.

The resolution of this puzzle lies in the presence of disorder. We shall see (Figure 2) that in the presence of static phase disorder (pinned vortices), the supercurrent profile can be pinned by disorder. The time-independent supercurrents can then penetrate into the sample over indefinitely large distances, limited only by the finite size of the sample. In fact, we find that dissipation only appears when supercurrents completely fill the sample. This mechanism gives a critical current (11) which is proportional to the area of the sample. The magnitude of this critical current agrees with experiments, using reasonable estimates of the parameters [18].

This paper is organized as follows. We will discuss the origin of disorder in the bilayer in Section 2. Then, in Section 3, we will introduce the phase Hamiltonian for the excitonic superfluid that describes the interlayer-coherent phase of the quantum Hall bilayer. In Section 4, we discuss

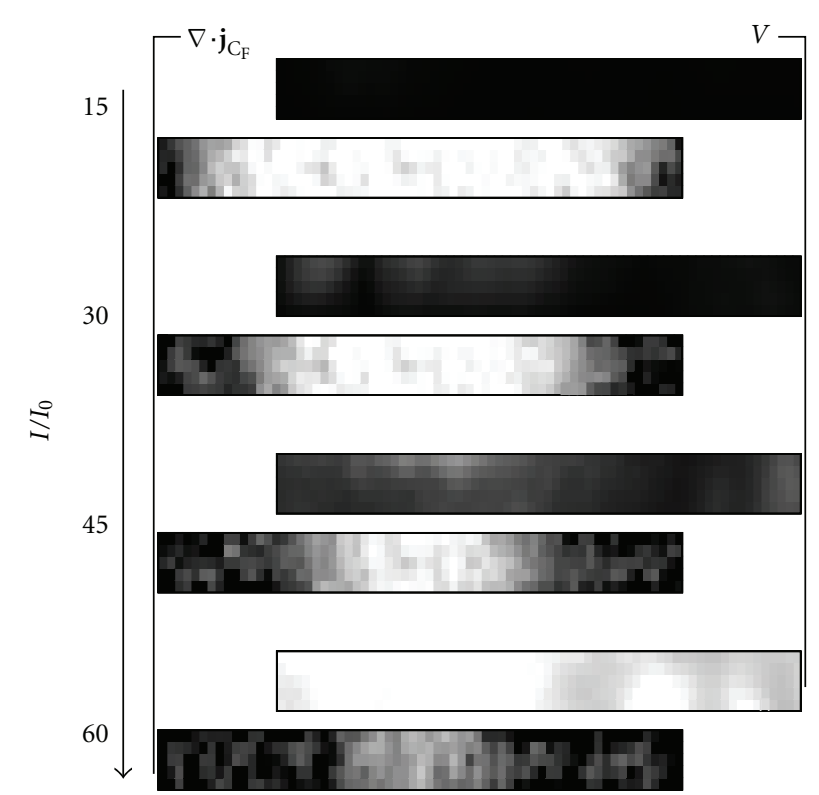

FIGURE 2: Spatial distribution of tunneling currents (left column) and interlayer voltages (right column), in a lattice model of $200 \times$ 20 sites, with current injection at the two lower corners. The injected counterflow currents are $I / I_{0}=15,30,45,60$ for the four pairs of plots. Dark colours, left column: high current. Dark colours, right column: low voltage. $t \xi^{2} / \rho_{s}=0.6$. Results are averaged over 500 realizations; tunneling currents are summed over blocks of $3 \times 3$ sites.

how quenched vortices in the superfluid affect the ground state of the system and its response to injected currents. Then, in Section 5, we present the results of a numerical simulation of the bilayer in the tunneling configuration to support the prediction of our theory. In the final section, we discuss how our picture can be used to interpret experiments for the bilayer in other configurations.

\section{Model of Disorder}

Weak disorder, such as a spatially varying tunneling splitting, does not affect the tunneling properties of the system dramatically [24]. A tunneling mechanism based on a disordered edge has been proposed by Rossi et al. [25] However, such a theory predicted linear scaling with the sample length but not its area.

We consider here a bilayer with charge disorder in the bulk. One common source for this disorder is the electrostatic potential due to disordered dopant layers. We expect the incompressible quantum Hall phase to occupy only a fraction of the sample, with the remainder occupied by puddles of compressible electron liquid. Thus, the incompressible phase forms a network of channels separating puddles of size $\xi \approx d_{d} \approx 200 \mathrm{~nm}$, the distance to the dopants. We suppose that the width of the channels is of the order of the magnetic length $\ell_{B} \sim 20 \mathrm{~nm}$. This coherent network model was first studied in the context of the quantum Hall bilayer by Fertig and Murthy [26]. 


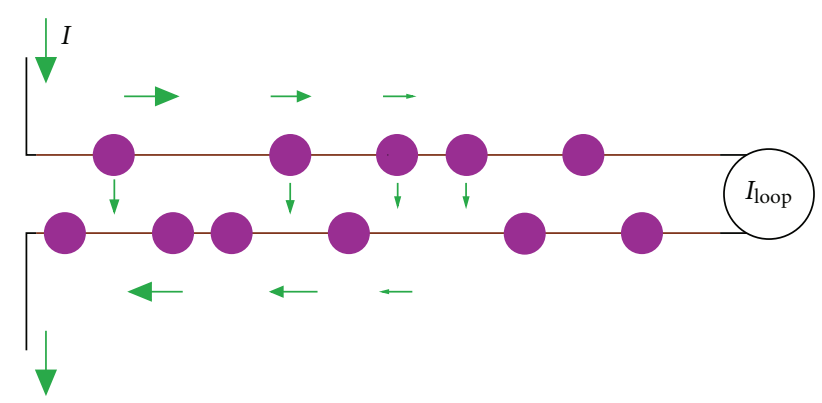

Figure 3: Schematic diagram of a counterflow experiment with a short circuit to complete current loop for counterflow. $I_{\text {loop }}$ measures current through the short circuit. Diagram depicts the Josephson regime where the loss of counterflow current through tunneling means that $I_{\text {loop }}=0$. The current-carrying region penetrates to the right as the injected current $I$ is increased, eventually reaching the other end at $I_{c}$.

In a quantum Hall superfluid, excess charge nucleates vortices in the exciton superfluid $[16,17,26-28]$. For a balanced bilayer with individual layer fillings $\nu_{1}=\nu_{2}=1 / 2$ these vortices are merons of charge $\pm e / 2$. (In an unbalanced bilayer, the charges are [29] $\pm e v_{1(2)}$.) In previous work [27], we have studied how the vortex density is determined by a competition between the superfluid energy cost of the vortex and the charging energy of each puddle. We found that the bilayer can be strongly disordered in the current experimental regimes. This suggests that the random field due to the pinned vortices has an exponentially decaying correlation function in space.

The above scenario provides a specific physical model for quenched vortices with short-ranged correlations in the exciton superfluid. The theory we present below depends on the existence of trapped fractional $e / 2$ charges to create these vortices but does not depend crucially on the details of the disorder distribution. Our results should be valid as long as the vortices are dense enough that their separation $(\sim \xi)$ is smaller than the clean tunneling length scale $\lambda_{J}(6)$.

\section{Phase Model}

In the previous section, we have outlined a model of disorder which induced quenched vortices in a quantum Hall state. To describe this exciton superfluid with quenched vortices, we start with an effective Hamiltonian for the phase $\theta$ of the superfluid. We separate out the component, $\theta^{0}$, of the phase field that is due to the quenched vortices. The remaining component, $\phi \equiv \theta-\theta^{0}$, would have no vorticity in the ground state but may acquire vorticity in the presence of injected currents and other external perturbations. It can be shown that the effective Hamiltonian can be written as a random field XY model

$$
H_{\mathrm{eff}}=\int\left[\frac{\rho_{s}}{2}(\nabla \phi)^{2}-t \cos \left(\phi+\theta^{0}\right)\right] d^{D} \mathbf{r},
$$

which describes the low-energy phase fluctuations of a bilayer containing pinned vortices. This form is a simple extension of the form for a clean model [8]. The first term describes the superfluid stiffness to phase twists, while the second describes the interlayer tunneling. We will assume that the quenched phase field $\theta^{0}$ has a correlation length of $\xi$.

In the Josephson regime, there is no quasiparticle flow at zero temperature. All currents are accounted for by superflow and coherent tunneling. The counterflow supercurrent density above the ground state, $\mathbf{j}_{\mathrm{CF}}$, and the interlayer tunneling current density, $J_{t}$, are related to the phase field by

$$
\mathbf{j}_{\mathrm{CF}}=\frac{e \rho_{s}}{\hbar} \nabla \phi, \quad J_{t}=\frac{e t}{\hbar} \sin \left(\phi+\theta^{0}\right) .
$$

A time-varying superfluid phase $\phi(t)$ gives rise to an interlayer voltage difference $V$ via the Josephson relation

$$
V=\frac{\hbar \dot{\phi}}{e}
$$

Therefore, a state with a finite interlayer current at zero interlayer voltage is time-independent, corresponding to a local minimum of the energy (2). The stationary equation is simply the continuity equation stating that the loss of counterflow current is accounted for by interlayer tunneling $\nabla \cdot \mathbf{j}_{\mathrm{CF}}=J_{t}$. This can be written as

$$
-\rho_{s} \nabla^{2} \phi+t \sin \left(\phi+\theta^{0}\right)=0 .
$$

All states with zero interlayer voltage obey this equation. The dependence on the injected current arises as the boundary conditions at the source and drain specifying the injected counterflow component $\mathbf{j}_{\mathrm{CF}}$. In terms of the phase field, this is a boundary condition on $\nabla \phi$.

We expect that the counterflow current injected at the boundary will decay into the sample because interlayer tunneling will recombine electrons and holes across the two layers, as depicted in Figure 3. In the clean case $\left(\theta^{0}=0\right)$, one expects $[20,30]$ the static solution to show all the injected counterflow current tunneling across the bilayer over a "Josephson length" of

$$
\lambda_{J}=\sqrt{\frac{\rho_{s}}{t}} .
$$

This length scale is estimated to be of the order of a few microns using realistic parameters.

Since the phase angle is compact, this implies a maximum injected current density of $\rho_{s}|\nabla \phi| \sim \pi \rho_{s} / \lambda_{J}$. For higher injected currents, phase slips enter and propagate through the system. This gives rise to a time-varying phase and hence a nonzero interlayer voltage via the Josephson relation (4).

Note that this picture of current penetration into the clean system gives a penetration depth as a microscopic length scale independent of the injected current. We will see below that the disordered system behaves qualitatively differently-the current can penetrate into an indefinitely large area of the system. The reason is that injected phase slips are pinned by disorder, and therefore, a static solution to (5) can persist to higher injected currents. In the next section, we will discuss this picture of pinning. 


\section{Pinned Superfluid}

We will now review the heuristic theory of pinning presented in our previous work [18] in order to provide the context to interpret our simulation results. The quenched vortices play a crucial role for the critical current. They pin any injected supercurrents and sustain dissipationless states. This is reminiscent of how disorder pins magnetic flux in superconductors [31-33] or charge in charge-density waves [34]. However, we emphasize that there is a significant difference in the bilayer compared with these other systems. In the superconductor, the depinning force arises from the Lorentz force on the flux lines due to the bulk transport current. In charge-density-wave systems, depinning originates from the electric field in the bulk which is an insulator when the charge density cannot slide. In the quantum Hall bilayer, depinning arises from the injected charge current which is applied only at the sample boundary. Thus, in this case, the critical current will depend on how the depinning "forces" are transmitted through the system. In such a geometry, it is not immediately obvious how the critical current $I_{c}$ would scale with the area of the whole sample.

We will borrow from the Fukuyama-Lee theory [34] of disordered charge density waves and the Imry-Ma theory [35] for ferromagnets in random fields. We recall the form of the ground states of the random field XY model, (2), in the weak disorder regime $\xi \ll \lambda_{J}$ relevant for the bilayer. In this regime, it is energetically costly for the phase $\phi$ to follow the random field $\theta^{0}$ which varies over the scale of the correlation length $\xi$. The ground state consists of domains of polarized phase. These domains cannot be arbitrarily large, because the energy cost of the mismatch between the phase and random field grows with the domain size. The energy cost for a phase twist that varies over the scale $l$ is $E_{s}(l) \sim$ $\rho_{s} l^{D-2}$ in $D$ dimensions. The typical tunneling energy of a polarized region of size $l$ is obtained by summing random energies in the range $\pm t \xi^{D}$ for its $(l / \xi)^{D}$ correlation areas, giving $E_{t}(l) \sim t \xi^{D}(l / \xi)^{D / 2}$. The phases will twist when $E_{t}(l)$ exceeds $E_{s}(l)$. Therefore, the ground state consists of domains of size $L_{\mathrm{d}}$ determined by

$$
E_{s}\left(L_{\mathrm{d}}\right) \sim E_{t}\left(L_{\mathrm{d}}\right) .
$$

This "Imry-Ma scale" for the domain is

$$
L_{\mathrm{d}} \sim\left(\frac{\rho_{s}}{t \xi^{D / 2}}\right)^{2 /(4-D)}=\left(\frac{\lambda_{J}^{2}}{\xi^{D / 2}}\right)^{2 /(4-D)} .
$$

In this ground state of polarized domains, the average coarse-grained phase over a domain is chosen such that the tunneling energy $H_{t}$ of each domain is minimized. Since $\delta H_{t} / \delta \phi(\mathbf{r})$ is the tunneling current at position $\mathbf{r}$, the total tunneling current over the domain vanishes.

In two dimensions, $L_{\mathrm{d}}=\lambda_{J}^{2} / \xi$. We see that in the experimentally relevant regime of $\lambda_{J} \gg \xi$, this new disorderinduced length scale is much larger than the Josephson length. This is the length scale controlling current penetration into the sample. However, as we see below, this should not be interpreted simply as a renormalized length scale for how far counterflow currents penetrate into the sample.
Consider now the effect of an injected counterflow which imposes a phase twist at the boundary. The phase will therefore twist away from its equilibrium configuration. We assume that the domain at the boundary remains polarized at short distances and so will rotate uniformly on the scale of $L_{\mathrm{d}}$. This generates a tunneling current which reduces the counterflow current. The residual counterflow currents will be transmitted further into the sample, causing the domains there to rotate in a similar way.

This picture allows us to average over each domain. The total tunneling current in a domain consists of a similar random sum to that for the tunneling energy, $E_{t}$, and is given by $I_{\mathrm{d}} f(\bar{\phi})$, where

$$
I_{\mathrm{d}}=\frac{e E_{t}\left(L_{\mathrm{d}}\right)}{\hbar}=\frac{e \rho_{s}}{\hbar} L_{\mathrm{d}}^{D-2}
$$

$\bar{\phi}$ is the deviation of the coarse-grained phase from its equilibrium value, and the range of $f(\bar{\phi})$ is typically $[-1,1]$. To minimize the region pushed out of equilibrium by the injected current, each domain will rotate so as to minimize the counterflow current transmitted into the sample. This maximizes the tunneling current and is achieved if we choose $|f| \sim 1$. Thus, we argue that forcing at a boundary leads to a self-organized critical state, in which the driven part of the system is saturated at the threshold $|f| \sim 1$. This means that the area $S_{t}$ of the system driven out of equilibrium to provide coherent tunneling is simply proportional to the number of domains necessary to carry the injected current $I$. Each domain can support a current of $I_{\mathrm{d}}$, and so

$$
\frac{S_{t}(I)}{L_{\mathrm{d}}^{D}} \approx \frac{I}{I_{\mathrm{d}}}
$$

The critical current is reached when all domains in the sample are saturated: $S=S_{t}\left(I_{c}\right)$ for a $2 \mathrm{D}$ sample of area $S$. Therefore, the critical current for a sample of area $S$ is

$$
I_{c} \sim I_{\mathrm{d}} \frac{S}{L_{\mathrm{d}}^{D}}=\frac{e \rho_{s}}{\hbar} \frac{S}{L_{\mathrm{d}}^{2}} .
$$

This formula also applies to the $1 \mathrm{D}$ case with $S$ being the sample length.

\section{Numerical Results}

We will now present numerical results to support the theory in the previous section. Our numerical results are obtained using the dissipative model

$$
-\lambda \dot{\phi}=\frac{\delta H_{\mathrm{eff}}}{\delta \phi}=-\rho_{s} \nabla^{2} \phi+t \sin \left(\phi+\theta^{0}\right),
$$

whose stationary solutions $\dot{\phi}=V=0$ are the local minima of (2). This is performed on a lattice model. The phase field $\theta_{i}^{0}$ at site $i$ is uncorrelated with the phase field at any other site. This corresponds to taking the lattice spacing to be the correlation length $\xi$ of the original continuum model. The natural unit of current is $I_{0}=e \rho_{s} / \hbar$. The results that we present below are the results for a $200 \times 20$ lattice, 
averaged over 500 realizations of the disorder. For this illustration, we take the ratio of the tunneling strength to the superfluid stiffness to be $t \xi^{2} / \rho_{s}=0.6$. This corresponds to a Josephson depth $\lambda_{J}$ of the order of a lattice spacing and a domain size $L_{\mathrm{d}}$ of 2 lattice spacings. Although this is not deep in the weak-disorder regime considered in the previous section, our results appear to support the conclusions in that section.

The boundary conditions for (2) are determined by the current flows through the sample [12]. We consider a tunneling geometry in which, as seen in Figure 2, a current $I_{t}$ is injected into the top layer at the bottom left corner and removed from the bottom layer at the bottom right corner. As already discussed in (1), the counterflow component of the currents corresponds to equal counterflow currents $I_{\mathrm{CF}}$ injected by both the electron source and drain.

The ground state of the system is found by evolving from a random state using the dissipative dynamics (12) with the boundary condition of no injected current. From (3), this corresponds to $\hat{\mathbf{n}} \cdot \nabla \phi=0$ everywhere on the boundary with $\hat{\mathbf{n}}$ being the normal to the boundary. To model the current injection in a tunneling experiment, we then slowly increase the counterflow current at the source and drain sites ( 1 and 2 ) to the final values $\left.\xi \hat{\mathbf{n}} \cdot \nabla \phi\right|_{1}=-\left.\xi \hat{\mathbf{n}} \cdot \nabla \phi\right|_{2}=I / I_{0}$. For the low values of the injected current $I$, the dynamics reach a static solution, corresponding to the Josephson regime with vanishing interlayer voltages. At higher currents, these time-independent solutions break down and the phase winds continuously in time. This corresponds to the breakdown of the d.c. Josephson regime and the appearance of a state with finite interlayer voltages.

We expect that the counterflow current injected at the boundary will decay into the sample, because interlayer tunneling will recombine electrons and holes across the two layers. We find that the manner in which this occurs is qualitatively different in clean and disordered bilayers. As mentioned in Section 1, the penetration depth of the injected current is simply the Josephson length $\lambda_{J}$ in the clean case. We see in Figure 2 that for the disordered case, the current penetrates further and further into the sample as we increase the injected current from the two ends. We see from the border of the regions with finite tunneling $\left(\nabla \cdot \mathbf{j}_{\mathrm{CF}} \neq 0\right)$ that the counterflow region increases linearly in area $\left(S_{t}\right)$ with the injected current. This is consistent with the prediction (10) for $S_{t}$ as a function of the injected current from our theory.

At a high enough injected current $\left(I / I_{0} \simeq 50\right)$, the current profiles from the contacts (lower left and right corners) will meet in the middle of the lattice. Beyond this point, further increases in current cannot be accommodated by coherent tunneling and an interlayer voltage develops.

We emphasize that this interpretation of the threshold for the breakdown of the stationary solutions is qualitatively different from the clean case. In the clean model, the breakdown can be understood in terms of the injection of phase solitons at the boundary $[20,36]$ when the injected current exceeds the superflow that can be supported by a static phase twist $|\nabla \phi| \sim \pi / \lambda_{J}$. These phase solitons propagate through the sample. Thus, the phase at any point varies in time, and the system develops an interlayer voltage by the a.c. Josephson effect. In this language, we can say that these injected solitons can be pinned by disorder so that stationary solutions exist even when there are many solitons in the system.

\section{Discussion}

We have so far focused our discussion on the bilayer in the tunneling geometry. Finally, we will discuss how two other experimental situations can be interpreted in our theory. The first setup is the transport in the bilayer in a counterflow geometry, where the source and drain contacts are on the same side of the bilayer, while the other end is short-circuited to allow the current to flow from the top layer to bottom without the need for tunneling. This is depicted schematically in Figure 3. This was first investigated by Tutuc et al. [22] and Kellogg et al. [23]. A large current ( $I_{\text {loop }}$ in figure) was found passing through the short circuit that join the top and bottom layers. This seems to imply that there is no leakage by tunneling across hundreds of microns. As we discussed in Section 1 for the case of the tunneling geometry, a realistic estimate of the tunneling rate based on a clean bilayer predicts that the injected current would have tunneled across the bilayer within a micron and that no current should remain at the far end.

In our theory, this situation can be simulated by solving (12) with injected counterflow current at one end only, say the left end of Figure 2. We expect the tunneling domains to saturate successively from this end, and the current profile is the same as that found in Figure 2 for this side of the sample. There will be no current flow on the right side. In other words, we expect to see zero current in the short-circuit loop $\left(I_{\text {loop }}=0\right)$ in the Josephson regime. As we increase the injected current $I$ to $I_{c}$, the current-carrying region reaches the other end of the sample. Any further currents will pass through the short circuit. For an ideal loop, we expect $I_{\text {loop }}=$ $I-I_{c}$. However, the short circuit itself should have a finite resistance. Therefore, the presence of a nonzero $I_{\text {loop }}$ implies a small interlayer voltage at the end of the sample. In the phase theory, the Josephson relation (4) means that the superfluid phase must wind in time. Thus, a static solution to (12) becomes impossible anywhere in the system, and the whole sample develops an interlayer voltage. We expect that the phase dynamics will be complex and chaotic. The nature of the steady state would depend on details of the damping mechanisms. This provides a zero-temperature picture of the counterflow geometry and is consistent with the recent experiments of Yoon et al. [37], in which the loop current $I_{\text {loop }}$ is negligible for tunnel currents below a critical value. A broadly similar scenario for these experiments has been recently suggested by Gilbert [38]. We should keep in mind that at finite temperatures, there may be in-plane resistances associated with the flow of vortices and thermally activated quasiparticles. Thermal effects have been considered by many authors [26, 39]. Most recently, Hyart and Rosenow have modeled this with a decoherence rate that crosses over from a thermally activated form at high temperatures to a power law form at lower temperatures [40]. 
The second situation we wish to discuss is the twoterminal measurements of zero-bias interlayer conductance, $G(0)$, by Finck et al. [19]. Within our theory, this could be interpreted as the dissipative regime (i.e., no static solutions for the phase) above the critical current. We note that these measurements were performed close to the phase boundary between the excitonic superfluid with interlayer coherence and the incoherent $v=1 / 2$ liquids. We expect the critical current to vanish near the phase boundary $[11,18]$. Therefore, it is easy to exceed the critical current in this region of the phase diagram. Then, we see in Figure 2 that a nonzero interlayer voltage develops across the whole sample, and the tunneling current exists over the whole sample. In other words, $G(0)$ should be proportional to the area of the sample, as seen by Finck et al. We point out that whereas this interpretation gives an intrinsic zero-temperature source of a finite conductance, there may be other sources of dissipation. Even below $I_{c}$ there could be a finite dissipation due to contact resistances and thermally activated vortex motion $[39,40]$. Fluctuations in the pinning energies could also lead to very weakly pinned regions in large samples [41], which may lead to dissipation below $I_{c}$ even at $T=0$.

In summary, we have presented a theory of the Josephson regime of coherent tunneling in a disordered quantum Hall bilayer with static pinned vortices. We find that in the tunneling geometry, there are two current-carrying regions emanating separately from the source and drain contacts. In these regions, coherent tunneling is saturated. All injected counterflow current is lost by tunneling by the edge of these regions. The area of the saturated region $S_{t}$ grows linearly with the injected current $I$. This linear relation is predicted by our theory and is confirmed by the numerical results presented here. This is analogous to the Bean critical state for flux penetration into a disordered superconductor.

This picture tells us that the system reaches the critical current when the whole sample is saturated with coherent tunneling. This results in a critical current that is extensive for sufficiently large samples that contain many domains of polarized phase. In contrast, the clean limit [12] sees area scaling for $I_{c}$ only for small samples (small compared to the Josephson length).

Theoretically, our results are qualitatively different from clean theories [12] because of the existence of these pinned polarized domains. The size $L_{\mathrm{d}}$ of these domains is a disorder-induced length scale that emerges in our theory (8). This scale has no counterpart in the clean system. It would, therefore, be useful if this length scale can be probed in experiments. We note that for the area-scaling formula (11) to apply, the sample should be large enough to include many complete domains. For sample dimensions smaller than $L_{\mathrm{d}}$, the system should cross over to a regime where $I_{c}$ scales with the square-root of the sample dimension [18]

$$
\begin{aligned}
I_{c} & \sim \frac{e \rho_{s}}{\hbar} \sqrt{\frac{L_{x}}{L_{\mathrm{d}}}} \frac{L_{y}}{L_{\mathrm{d}}} \quad\left(\text { quasi-1D: } L_{x} \ll L_{\mathrm{d}} \ll L_{y}\right) \\
& \sim \frac{e \rho_{s}}{\hbar} \sqrt{\frac{L_{x} L_{y}}{L_{\mathrm{d}}^{2}}} \quad\left(\text { for } L_{x}, L_{y} \ll L_{\mathrm{d}}\right) .
\end{aligned}
$$

This crossover provides an experimental probe of the domain size $L_{\mathrm{d}}$.

\section{Acknowledgments}

The authors thank P. B. Littlewood for helpful discussions. This work was supported by EPSRC-GB (EP/C546814/01) and Science Foundation Ireland (SFI/09/SIRG/I1592).

\section{References}

[1] S. Q. Murphy, J. P. Eisenstein, G. S. Boebinger, L. N. Pfeiffer, and K. W. West, "Many-body integer quantum Hall effect: evidence for new phase transitions," Physical Review Letters, vol. 72, no. 5, pp. 728-731, 1994.

[2] T. S. Lay, Y. W. Suen, H. C. Manoharan, X. Ying, M. B. Santos, and M. Shayegan, "Anomalous temperature dependence of the correlated $v=1$ quantum Hall effect in bilayer electron systems," Physical Review B, vol. 50, no. 23, pp. 17725-17728, 1994.

[3] B. I. Halperin, "Theory of the quantized hall conductance," Helvetica Physica Acta, vol. 56, p. 75, 1983.

[4] H. A. Fertig, "Energy spectrum of a layered system in a strong magnetic field," Physical Review B, vol. 40, no. 2, pp. 10871095, 1989.

[5] J. P. Eisenstein and A. H. MacDonald, "Bose-Einstein condensation of excitons in bilayer electron systems," Nature, vol. 432, no. 7018, pp. 691-694, 2004.

[6] X. G. Wen and A. Zee, "Neutral superfluid modes and magnetic monopoles in multilayered quantum Hall systems," Physical Review Letters, vol. 69, no. 12, pp. 1811-1814, 1992.

[7] X. G. Wen and A. Zee, "Superfluidity and superconductivity in double-layered quantum Hall state," International Journal of Modern Physics B, vol. 17, no. 25, pp. 4435-4446, 2003.

[8] X. G. Wen and A. Zee, "Tunneling in double-layered quantum Hall systems," Physical Review B, vol. 47, no. 4, pp. 2265-2270, 1993.

[9] Z. F. Ezawa and A. Iwazaki, "Lowest-Landau-level constraint, Goldstone mode, and Josephson effect in a double-layer quantum Hall system," Physical Review B, vol. 48, no. 20, pp. 15189-15197, 1993.

[10] L. Tiemann, W. Dietsche, M. Hauser, and K. von Klitzing, "Critical tunneling currents in the regime of bilayer excitons," New Journal of Physics, vol. 10, Article ID 045018, 2008.

[11] L. Tiemann, Y. Yoon, W. Dietsche, K. von Klitzing, and W. Wegscheider, "Dominant parameters for the critical tunneling current in bilayer exciton condensates," Physical Review B, vol. 80, no. 16, Article ID 165120, 2009.

[12] J.-J. Su and A. H. MacDonald, "Critical tunneling currents in quantum Hall superfluids: pseudospin-transfer torque theory," Physical Review B, vol. 81, no. 18, Article ID 184523, 11 pages, 2010.

[13] I. B. Spielman, J. P. Eisenstein, L. N. Pfeiffer, and K. W. West, "Resonantly enhanced tunneling in a double layer quantum Hall ferromagnet," Physical Review Letters, vol. 84, no. 25, pp. 5808-5811, 2000.

[14] J. P. Eisenstein, "Evidence for spontaneous interlayer phase coherence in a bilayer quantum Hall exciton condensate," Solid State Communications, vol. 127, no. 2, pp. 123-130, 2003.

[15] R. L. Jack, D. K. K. Lee, and N. R. Cooper, "Dissipation and tunneling in quantum Hall bilayers," Physical Review Letters, vol. 93, no. 12, Article ID 126803, 4 pages, 2004. 
[16] A. Stern, S. M. Girvin, A. H. MacDonald, and N. Ma, "Theory of interlayer tunneling in bilayer quantum Hall ferromagnets," Physical Review Letters, vol. 86, no. 9, pp. 1829-1832, 2001.

[17] L. Balents and L. Radzihovsky, "Interlayer tunneling in double-layer quantum Hall pseudoferromagnets," Physical Review Letters, vol. 86, no. 9, pp. 1825-1828, 2001.

[18] P. R. Eastham, N. R. Cooper, and D. K.K. Lee, "Critical supercurrents and self-organization in quantum Hall bilayers," Physical Review Letters, vol. 105, no. 23, Article ID 236805, 4 pages, 2010.

[19] A. D. K. Finck, A. R. Champagne, J. P. Eisenstein, L. N. Pfeiffer, and K. W. West, "Area dependence of interlayer tunneling in strongly correlated bilayer two-dimensional electron systems at $\nu_{\mathrm{T}}=1$," Physical Review B, vol. 78, no. 7, Article ID 075302, 5 pages, 2008.

[20] D. V. Fil and S. I. Shevchenko, "Josephson vortex motion as a source for dissipation of superflow of e-h pairs in bilayers," Journal of Physics Condensed Matter, vol. 21, no. 21, Article ID 215701, 2009.

[21] M. Abolfath, A. H. MacDonald, and L. Radzihovsky, "Critical currents of ideal quantum Hall superfluids," Physical Review $B$, vol. 68, no. 15, Article ID 155318, 16 pages, 2003.

[22] E. Tutuc, M. Shayegan, and D. A. Huse, "Counterflow measurements in strongly correlated GaAs hole bilayers: evidence for electron-hole pairing," Physical Review Letters, vol. 93, no. 3, Article ID 036802, 4 pages, 2004.

[23] M. Kellogg, J. P. Eisenstein, L. N. Pfeiffer, and K. W. West, "Vanishing Hall resistance at high magnetic field in a doublelayer two-dimensional electron system," Physical Review Letters, vol. 93, no. 3, Article ID 036801, 4 pages, 2004.

[24] O. G. C. Ros and D. K. K. Lee, "Effect of disorder and electron-phonon interaction on interlayer tunneling current in quantum Hall bilayer," Physical Review B, vol. 81, no. 7, Article ID 075115, 7 pages, 2010.

[25] E. Rossi, A. S. Núñez, and A. H. MacDonald, "Interlayer transport in bilayer quantum Hall systems," Physical Review Letters, vol. 95, no. 26, Article ID 266804, 4 pages, 2005.

[26] H. A. Fertig and G. Murthy, "Coherence network in the quantum Hall bilayer," Physical Review Letters, vol. 95, no. 15, Article ID 156802, 4 pages, 2005.

[27] P. R. Eastham, N. R. Cooper, and D. K. K. Lee, "Vortex states of a disordered quantum Hall bilayer," Physical Review B, vol. 80, no. 4, Article ID 045302, 5 pages, 2009.

[28] M. M. Fogler and F. Wilczek, "Josephson effect without superconductivity: realization in quantum Hall bilayers," Physical Review Letters, vol. 86, no. 9, pp. 1833-1836, 2001.

[29] B. Roostaei, K. J. Mullen, H. A. Fertig, and S. H. Simon, "Theory of activated transport in bilayer quantum Hall systems," Physical Review Letters, vol. 101, no. 4, Article ID 046804, 4 pages, 2008.

[30] P. Bak, "Commensurate phases, incommensurate phases and the devil's staircase," Reports on Progress in Physics, vol. 45, no. 6, pp. 587-629, 1982.

[31] M. Tinkham, Introduction to Superconductivity, McGraw-Hill, New York, NY, USA, 1996.

[32] A. I. Larkin and Y. N. Ovchinnikov, "Pinning in type II superconductors," Journal of Low Temperature Physics, vol. 34, no. 3-4, pp. 409-428, 1979.

[33] V. M. Vinokur and A. E. Koshelev, "Collective flux pinning in extended Josephson junctions," Soviet Physics JETP, vol. 70, p. $547,1990$.

[34] H. Fukuyama and P. A. Lee, "Dynamics of the charge-density wave-I. Impurity pinning in a single chain," Physical Review $B$, vol. 17 , no. 2 , pp. 535-541, 1978.
[35] Y. Imry and S. K. Ma, "Random-field instability of the ordered state of continuous symmetry," Physical Review Letters, vol. 35, no. 21, pp. 1399-1401, 1975.

[36] P. B. Littlewood and T. M. Rice, "Metastability of the Q vector of pinned charge- and spin-density waves," Physical Review Letters, vol. 48, no. 1, pp. 44-47, 1982.

[37] Y. Yoon, L. Tiemann, S. Schmult, W. Dietsche, K. von Klitzing, and W. Wegscheider, "Interlayer tunneling in counterflow experiments on the excitonic condensate in quantum Hall bilayers," Physical Review Letters, vol. 104, no. 11, Article ID 116802, 4 pages, 2010.

[38] M. J. Gilbert, "Finite-temperature pseudospin torque effect in graphene bilayers," Physical Review B, vol. 82, no. 16, Article ID 165408, 12 pages, 2010.

[39] D. A. Huse, "Resistance due to vortex motion in the $v=1$ bilayer quantum Hall superfluid," Physical Review B, vol. 72, no. 6, Article ID 064514, 4 pages, 2005.

[40] T. Hyart and B. Rosenow, "Quantitative description of Josephson-liketunneling in $\nu_{\mathrm{T}}=1$ quantum Hall bilayers," preprint, http://arxiv.org/abs/1011.5684v1.

[41] S. N. Coppersmith, "Phase slips and the instability of the Fukuyama-Lee-Rice model of charge-density waves," Physical Review Letters, vol. 65, no. 8, pp. 1044-1047, 1990. 

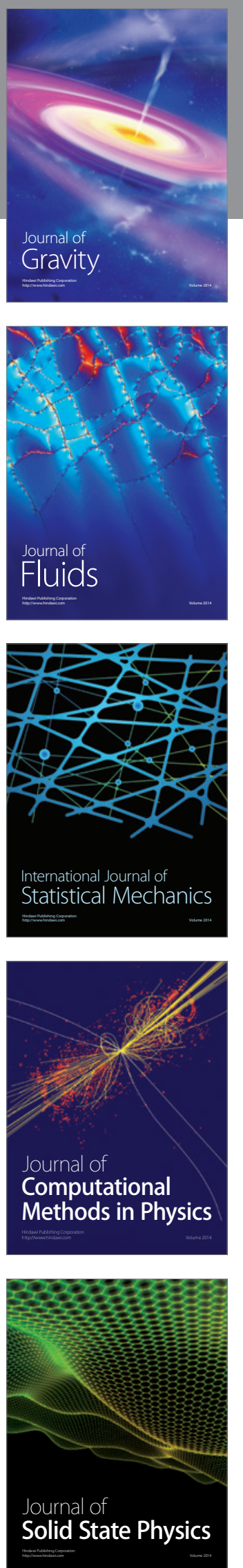

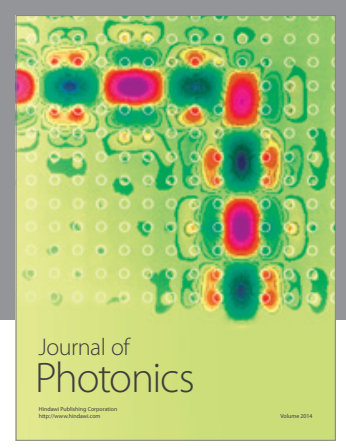

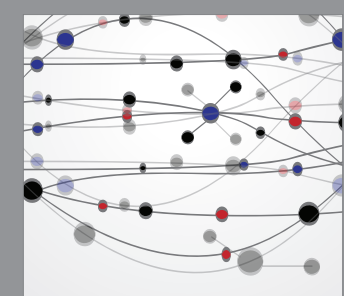

The Scientific World Journal
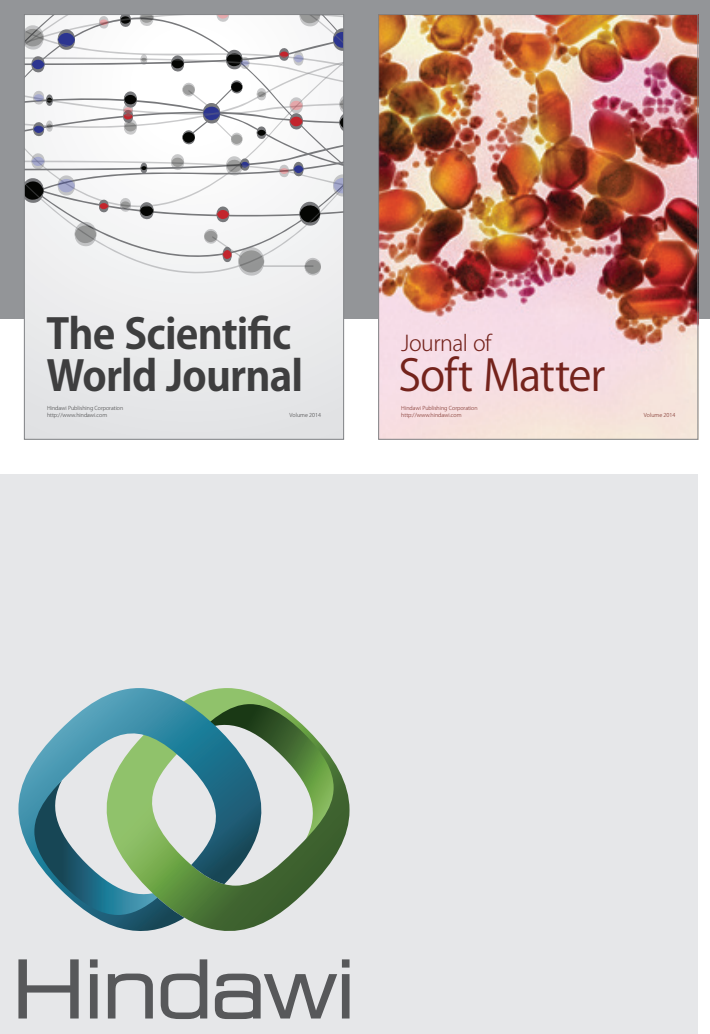

Submit your manuscripts at

http://www.hindawi.com
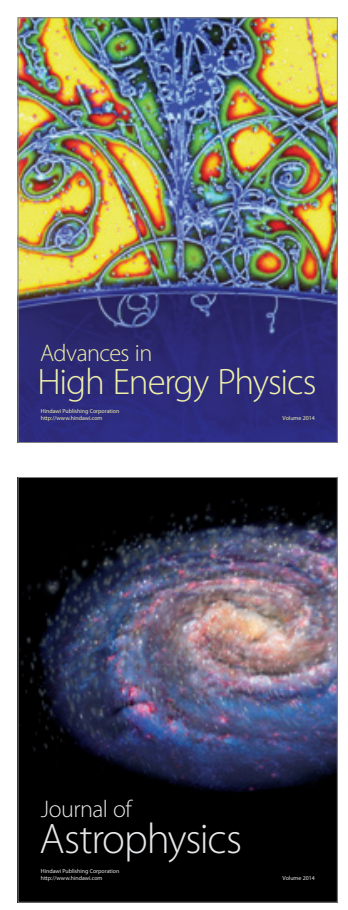
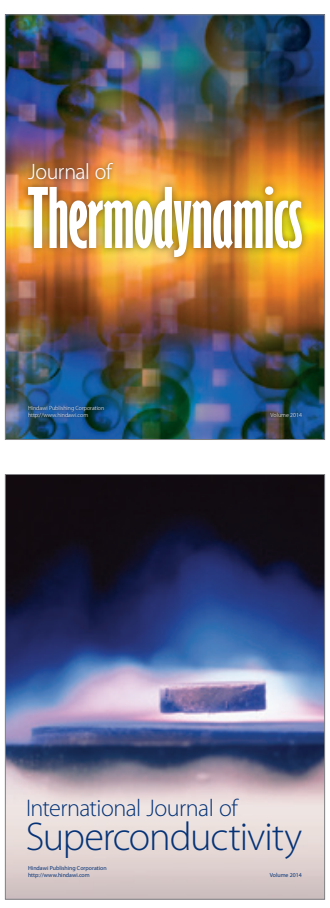
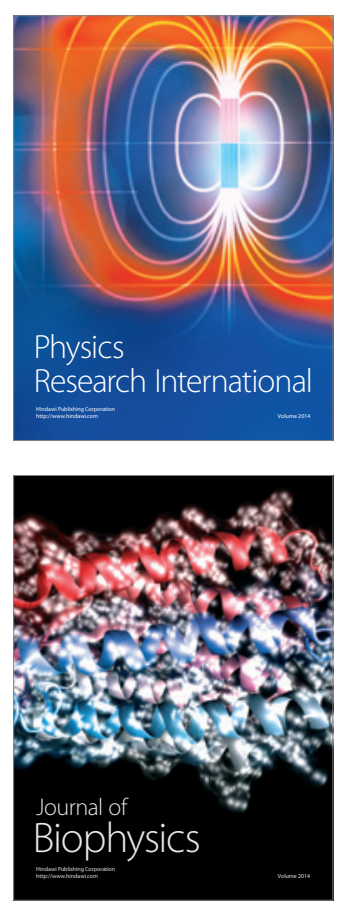
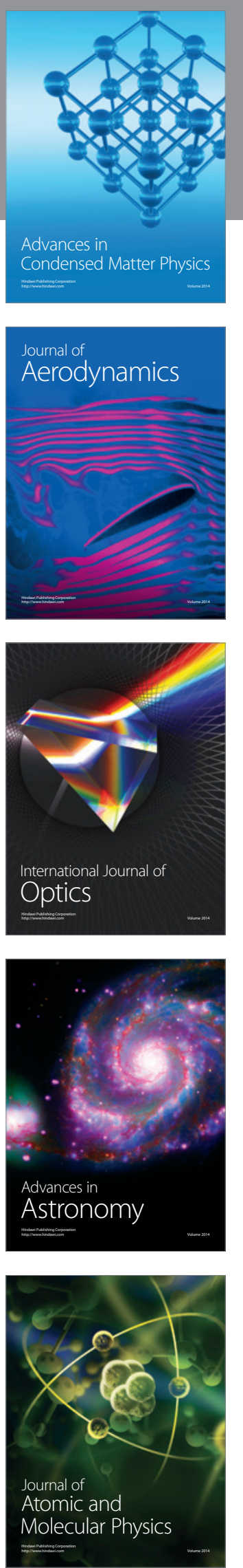